\title{
Spondyloarthritis-Patienten tun sich schwer, ihren Rückenschmerz einzuordnen
}

Klagen Patienten mit axialer Spondyloarthritis (axSpA) über Schmerzen, stellt sich immer die Frage: Ist dies auf strukturelle Folgeschäden, wie Ankylosen, zurückzuführen, oder ist die Rheumaerkrankung entzündlich aktiv? Dies hätte durchaus therapeutische Konsequenz.

Da Entzündungswerte nicht immer wegweisend sind - vor allem bei Psoriasisarthritis -, wäre es praktisch, wären Patienten selbst in der Lage, die beiden Schmerzformen zu unterscheiden. Einer niederländischen Studie von Dr. Anne Kampman und Kollegen zufolge ist das wohl nicht der Fall. Die Rheumatologin vom Medisch Center Leeuwarden stellte ihre Daten beim Jahreskongress der European League Against Rheumatism (EULAR) vor.

In die Beurteilung der axSpAKrankheitsaktivität, die in der Regel mit dem Bath Ankylosing Spondylitis Disease Activity Index (BASDAI) oder dem Ankylosing Spondylitis Disease Activity Score (ASDAS) erfasst wird, gehen überwiegend, beim BASDAI ausschließlich, subjektive Angaben der Patienten ein. Unter anderem zu Schmerzen. Ein Teil der Patienten weist daher, trotz antientzündlicher Therapie, konstant erhöhte AktivitätsScores auf.

\section{Schwierige Einordnung}

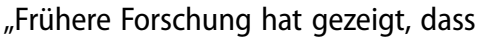
auch andere Schmerzmechanismen, wie eine zentrale Sensibilisierung, eine Rolle bei dem Schmerz, den Patienten erfahren, spielen können", räumte Kampman ein. Doch die waren nicht Gegenstand der Querschnittsanalyse, die die Wissenschaftler im Rahmen einer prospektiven Langzeitbeobachtungsstudie der Groningen Leeuwarden Axial Spondyloarthritis (GLAS)-Kohorte vornahmen. Zunächst wurden die Patienten aufgefordert, das Ausmaß ihrer Schmerzen in Nacken, Rücken oder Hüften auf einer numerischen Analogskala anzugeben. Dann vermerkten sie auf einer weiteren, in welchem Maße die Schmerzen ihrer Meinung nach entzündlich oder strukturell verursacht waren.

\) Die Bildgebung passte bei Patienten mit vermuteter entzündlicher Schmerzursache zu ihrer Einschätzung

Von 688 berücksichtigten Patienten sahen sich drei Viertel nicht in der Lage, dies zu differenzieren. $15 \%$ entschieden sich für eine entzündliche, zehn Prozent für eine mechanische Ursache.

\section{Korrelationen mit Anamnese und klinischen Befunden}

Soweit Patienten eine Differenzierung vornahmen, korrelierte dies weitgehend mit Anamnese und klinischen Befunden. Patienten, die glaubten, ihre Beschwerden seien strukturell bedingt, waren mit durchschnittlich rund 49 Jahren signifikant und im Mittel neun Jahre älter als die, die aufgrund ihrer subjektiven Entscheidung der Entzündungsgruppe zugeordnet wurden.

Patienten in der StrukturschadenGruppe waren mit durchschnittlich 28 gegenüber 12 Jahren bereits länger krank. Entsprechend schlechter waren auch die meisten ihrer Bewegungsausmaße, vor allem Hinterhaupt-Wand-
Abstand und Halswirbelsäulenrotation. Die Unterschiede beim modifizierten Schober-Test waren nicht signifikant.

\) Patienten können nicht zwischen strukturellen Folgeschäden und Entzündungsaktivität unterscheiden

Patienten in der Entzündungsgruppe erzielten signifikant höhere Scores bei ihrer eigenen subjektiven globalen Krankheitsaktivitäts-Einschätzung und beim ASDAS. In den floss der CRP-Wert ein, der allerdings für sich genommen in beiden Gruppen ähnlich war. Unterschiede beim BASDAl ergaben sich nur bei der Bewertung der Schmerzen in Nacken, Rücken und Hüften, nicht aber beim Gesamt-Score.

Die Bildgebungsbefunde passten bei Patienten mit vermuteter entzündlicher Schmerzursache übrigens durchaus zu ihrer Einschätzung. Kampman: „Sie hatten auch häufiger die Diagnose einer nicht-radiologischen axialen Spondyloarthritis." $40 \%$ waren das, in der StrukturschadenGruppe aber nur $13 \%$.

Hinweis des Verlags. Der Verlag bleibt in Hinblick auf geografische Zuordnungen und Gebietsbezeichnungen in veröffentlichten Karten und Institutsadressen neutral.

rheuma plus $2021 \cdot 20: 200$ https://doi.org/10.1007/s12688-02100456-5

○) Springer-Verlag GmbH Austria, ein Teil von Springer Nature 2021 